\title{
Effective field theory of Inflation in Hořava-Lifshtiz gravity
}

\author{
Shun Arai ${ }^{* \dagger}$ \\ Department of Physics and Astrophysics, Nagoya University, Nagoya 464-8602, Japan, \\ E-mail: arai.shunda.mbox.nagoya-u.ac.jp
}

\begin{abstract}
We study the evolution of primordial power spectra during inflation in the context of Hořava Lifshitz gravity. In this context, scalar perturbations are generally divided by 2 different d.o.f ; an inflaton fluctuation and a scalar graviton due to Lorentz invariance violation. However, we reveal that only the inflaton fluctuation contributes to a primordial curvature perturbation because the scalar graviton acquires its mass much larger than Hubble scale. Besides, the curvature perturbation becomes adiabatic, which is consistent with current observations of the Cosmic Microwave Background. In addition we find that a primordial gravitational waves provide a direct evidence of Lorentz invariance violation.
\end{abstract}

The 3rd International Symposium on" Quest for the Origin of Particles and the Universe" 5-7 January 2017

Nagoya University, Japan

* Speaker.

${ }^{\dagger}$ A footnote may follow. 


\section{Inflation as a probe for LIV}

Inflation is one of the most attractive phenomena in cosmology. Historically, inflation was firstly introduced to solve the cosmological problems [四, but inflation can also generate initial condition of the universe from quantum fluctuations that make seeds of cosmic structures. In this sense, we will expect that abundant imprints of high energy physics remains in cosmological observables. In this article, we would like to focus on the imprints Lorentz invariance in the early time of the universe. Lorentz invariance (LI) is one of the most fundamental symmetry in modern physics respected by a lot of fundamental theories. We would like to discuss how observables in inflationary epoch carry the information of Lorentz invariance violation (LIV) at present. In a theoretical point view, LIV is also adequate to interest us. Actually, Hořava-Lifshitz gravity [] has been developed recently as a counter candidate of quantum gravity instead of string theory. The main aim of our study is to grasp LIV features in inflationary universe and to pioneer the search of LIV phenomena in the cosmological context. More specifically, what we do in this article is that we investigate the features of primordial fluctuations on an inflation model with Hořava-Lifshtiz gravity and compute two main observables; tensor to scalar ratio $r$ and tensor tilt $n_{t}$. Usually, $r$ and $n_{t}$ are given by

$$
r=16 c_{s} \varepsilon
$$

where $c_{s}$ is a sound speed of an inflaton, $\varepsilon \equiv-\dot{H} / H^{2}$, and $H$ is the Hubble parameter. Then a consistency relation between scalar and primordial gravitational waves are given by

$$
n_{t}=-\frac{r}{8 c_{s}} .
$$

These observables can be tested by CMB observations[[]]. Besides, there are future proposals to realize direct detection of primordial gravitational waves such as eLISA[四] and DECIGO[G]. In prior to these observations, it is meaningful to provide how LIV features are imprinted in primordial fluctuations. In the next section we briefly summarize what we find.

\section{EFT treatment of scalar graviton}

Hořava-Lifsihtz gravity has two different properties from General Relativity; the anisotropic scaling law and the foliation-preserving diffeomorphism given as follows respectively:

$$
t \rightarrow b^{z} t, \mathbf{x} \rightarrow b \mathbf{x}, t \rightarrow \tilde{t}=\tilde{t}(t), \mathbf{x} \rightarrow \tilde{\mathbf{x}}=\tilde{\mathbf{x}}(\mathbf{x}, t) .
$$

Here $z$ denotes a critical exponent of time in ultraviolet limit. $z=1$ makes no difference from General Relativity. However in Hořava-Gravity $z$ is generally larger than 1 to permit to add higher spatial derivatives to keep the anisotropic scaling law. This is crucial to respect renormalizability for quantum correction of graviton self interactions. Hereafter we set $z=3$ for simplicity. In more detail discussion please see reviews [G, 团]. Due to the breakdown of general covariance, a new scalar d.o.f, scalar graviton, becomes dynamical. The previous studies shows cosmological perturbations on fixed backgrounds $[\mathbb{[}, \mathbf{Q}, \mathbf{U}, \mathbf{0}]$, but the consistency relation between scalar and primordial gravitational waves given by Eqs. (‥2) have yet to be fully investigated. We build an inflation model that includes interactions between the inflaton and the scalar graviton. Consequently, we 
find that the scalar graviton get decoupled from inflaton before Hubble crossing. This is because the scalar graviton gains its mass, $m_{K}$, which is given by

$$
m_{K}=\sqrt{\frac{M_{P}}{M_{L I V}}} H \gg H
$$

where $M_{L I V}$ is a typical scale of $\mathrm{LIV}^{1}$ and thus the scalar graviton is already decoupled from the inflaton fluctuation. As a result, the power spectrum of a gauge invariant curvature perturbation $\zeta$ is determined only by inflaton and we obtain

$$
\mathscr{P}_{\zeta} \propto \frac{1}{\varepsilon} \frac{M_{L I V}}{M_{P}}\left(\frac{H}{M_{L I V}}\right)^{3 / z-1} .
$$

Here are some comments for the spectrum. First of all, $\zeta$ is adiabatically conserved due to decoupling of the scalar graviton. This indicates that the scalar perturbations during inflation we can put the effect of the scalar graviton describing with an effective field theory approach. Observational points of view, the two following things are important. The one is that the latest observations of CMB support adiabatic initial condition of inflation. Therefore, our result is still consistent with the observations. The other is that flatness of the spectrum. Due to a scaling invariance at $z=3, \mathscr{P}_{\zeta}$ becomes scale invariant spectrum. Note that this result we don't investigate a running correction of slow-roll parameters.

\section{PGW as a smoking gun of LIV}

Let's consider the primordial gravitational waves (PGW). P.Creminelli et.al[ए]] shows that Eq. (ㄴ.2) generally preserves in a inflation model with general covariance. However in HořavaLifshitz gravity, we obtain a modified cosistency relation as

$$
n_{t}=-\frac{z-3}{z} \varepsilon
$$

As we mentioned, we now consider the case $z=3$ so that we find that Eq. $\square .2$ can be violated as

$$
n_{t}=0
$$

which means PGW provides a direct evidence to indicate LIV in the inflationary epoch. Recently it has been reported that G-inflation [ए2] or k-inflation [ए[3] can provide a violating consistency relation, all of which break null energy condition. What we have to stress here is that our results is quite different from that reports because we still respect the null energy condition but the consistency relation is disrespected. This is purely due to LIV in Hořava-Lifshitz gravity. Therefore the direct detection of PGW can be a smoking gun of Lorentz symmetry.

\section{Conclusion}

We have studied the evolution of primordial power spectra during inflation in the space-time described by Hořava-Lifshitz gravity. During inflaton not only the inflaton fluctuation but also

\footnotetext{
${ }^{1}$ We assume that $M_{L I V}$ is sub-Planckian scale.
} 
the scalar graviton are dynamical. However, the scalar graviton become massive and is decoupled from inflaton. As a result, gauge invariant curvature perturbation is determined only by inflaton. Considering together that inflaton is in WKB phase before Hubble crossing, the power spectrum of the curvature perturvation becomes adiabatic, which is consistent with current observations of the CMB. In addition, we have found that a primordial gravitational waves provide a direct evidence of Lorentz symmetry violation. We will submit these results in this article soon.

\section{Acnknowledgements}

SA is in part supported by MEXT Grant-in- Aid for Scientific Research on Innovative Areas, No. 15H05890. In KMI 2017 international conference. We appreciate E.Barausse and N. Takeda for useful discussions. We thank all organizers of KMI2017.

\section{References}

[1] A. H. Guth, "Inflationary universe: A possible solution to the horizon and flatness problems," Phys. Rev. D 23, 347 (1981)

[2] P. Hořava, “Quantum Gravity at a Lifshitz Point,” Phys. Rev. D 79, 084008 (2009)

[3] P. A. R. Ade et al. [Planck Collaboration], "Planck 2015 results. XX. Constraints on inflation," doi:10.1051/0004-6361/201525898

[4] P. Amaro-Seoane et.al, "Low-frequency gravitational wave science with eLISA/NGO," Class. Quant. Grav. 29, 124016 (2012) doi:10.1088/0264-9381/29/12/124016

[5] N. Seto, S. Kawamura, and T. Nakamura, "Possibility of Direct Measurement of the Acceleration of the Universe Using 0.1 Hz Band Laser Interferometer Gravitational Wave Antenna in Space,” Phys. Rev. Lett. 87, 221103 (2001) doi: 10.1103/PhysRevLett.87.221103

[6] S. Mukohyama, "Hořava-Lifshitz Cosmology: A Review," Class. Quant. Grav. 27, 223101 (2010) doi:10.1088/0264-9381/27/22/223101

[7] D. Blas, O. Pujolas and S. Sibiryakov, "Models of non-relativistic quantum gravity: The Good, the bad and the healthy," JHEP 1104, 018 (2011)

[8] K. Izumi, T. Kobayashi, and S. Mukohyama, “Non Gaussianity from Lifshitz Scalar,” JCAP 1010, 031 (2010)

[9] T. Kobayashi, Y. Urakawa and M. Yamaguchi, "Large scale evolution of the curvature perturbation in Hořava-Lifshitz cosmology,’ JCAP 0911, 015 (2009) doi:10.1088/1475-7516/2009/11/015

[10] C. Armendariz-Picon, N. F. Sierra and J. Garriga, "Primordial Perturbations in Einstein-Aether and BPSH Theories," JCAP 1007, 010 (2010)

[11] P. Creminelli, J. Gleyzes, J. Norena and F. Vernizzi, "Resilience of the standard predictions for primordial tensor modes," Phys. Rev. Lett. 113, no. 23, 231301 (2014) doi:10.1103/PhysRevLett.113.231301

[12] T. Kobayashi, M. Yamaguchi, and J. Yokoyama, "G - inflation: inflation dirven vy the Galileon field," Phys. Rev. Lett. 105, 231302 (2010) doi:10.1103/PhysRevLett.105.231302

[13] C. Armendariz-Picon, T. Damour, and V. Mukhanov, "k-Inflation,” Phys. Rev. B 458, 209-218 (1999) doi: 10.1016/S0370-2693(99)00603-6 